\title{
RETRACTED ARTICLE: Role of aminolevulinic acid synthase 1 in doxorubicin-induced oxidative stress to the ardiomyocyte
}

\section{Zuoyan Wang $^{1} \cdot$ Junyi Gao ${ }^{1} \cdot$ Haobo Teng ${ }^{1} \cdot$ Jianjun Peng ${ }^{1}$}

Received: 24 June 2019 / Accepted: 19 December 2019 / Published online: 7 January 2020

(C) Springer-Verlag GmbH Germany, part of Springer Nature 2020

This article is retracted by the Editor-in-Chief at the request of the corresponding author who stated that the result presented in Figure 3A for rALAS1 group was duplicated from another figure. Therefore the data and conclusions of this study are unreliable. All authors agree to this retraction.

The online version of this article contains the full text of the retracted article as electronic supplementary material.

Electronic supplementary material The online version of this article (https://doi.org/10.1007/s00210-019-01799-9) contains supplementary material, which is available to authorized users.

Jianjun Peng pjj0630@163.com

1 Department of Cardiology, Beijing Shijitan Hospital, Capital Medical University, No.10 Tieyi Road, Haidian District, Beijing 100038, China 